\title{
MAC Implemented using TCP, UDP and HTTP Protocols in Congestion Control
}

\author{
Tarannum Khan ${ }^{1}$, Sachin Upadhyay ${ }^{2}$ \\ Student, Computer Science and Engineering, Alpine Institute of Technology, Ujjain, India ${ }^{1}$ \\ Assistant Professor, Computer Science and Engineering, Alpine Institute of Technology, Ujjain, India ${ }^{2}$
}

\begin{abstract}
Congestion is necessary concern that can occur within the packet switched network. Here discussion on Congestion control gives the approach and appliances to control the problem of congestion and either avoid congestion from happening or eliminate the congestion once it has taken place. This paper summarizes the different category provided through the congestion control. Here It also discussed on how TCP utilize congestion and manages to improve congestion or avoid congestion in the network. In this paper we proposed that all the activity which we are managing by our proposed scheme is assure about the reliability aspects due to the connection oriented TCP services and the previous working of the "multicast congestion avoidance" (MCA) lower version protocol has been implemented as UDP connection-less services. Hence reliability will be increased significantly.
\end{abstract}

Keywords: Congesion Control; Congesion Control Algorithm, "Multicast Congestion Avoidance" (MCA).

\section{INTRODUCTION}

Congestion control over the network, for all the types of the media traffic, has been active area of the research within the last decade [1]. This is due to the successful growth in audiovisual traffic of digital convergence. There exists an expansion of network applications constructed on its capability of streaming media both in actual-time or on call for which includes video streaming and conferencing, the voice over IP which is known as (VoIP), and the video on demand which is known as $(\mathrm{VoD})$. The wide variety of users for those network programs is constantly growing as a result resulting in congestion. In networks, the packet loss can occur due to transmission errors, however most regularly because of congestion. TCP's congestion control mechanism reacts to packet loss by using losing the number of unacknowledged statistics segments allowed within the n/w. TCP flows with comparable round-trip times (RTTs) that shares a common bottleneck to lessen their rate in order that the accessible bandwidth can be continuous, disbursed equally amongst them. Not all network applications use TCP and therefore do not allow the same concept of fairly allocation the available bandwidth. Thus, the outcome of the unfairness of non-TCP applications doesn't have a large amount of impact as most of the traffic inside the network uses the protocols which is TCP-based. However, the quantity of audio/video streaming usage inclusive of Internet audio and video players, video conferencing and analogous forms of actual-time programs is frequently growing and it is soon anticipated that there could be a growth in the share of non-TCP traffic. In analysis of the fact that such applications usually don't amalgamate TCP compatible congestion control mechanisms; the network application treats the tough TCP-flows in a difficult manner. Every part of the TCP-flows decrease their data rates in an effort to break up the problem of congestion, where the non-TCP flows is maintain to send with their novel rate.. This notably unfair condition will result in starvation of TCP-traffic i.e., congestion collapse [2], [3], which describes the disagreeable situation where the accessible bandwidth in a network is almost entirely occupied by packets which are discarded due to the congestion earlier than they attain their destination. For this motive, it's far ideal to define suitable congestion manage mechanisms for non-TCP traffic, that are compatible with the rate-adaptation mechanism of TCP .These mechanisms ought to make non-TCP programs TCP-friendly, and hence product in an honest distribution of the bandwidth. Unicast is the one to one type of communication inside the network where multicast istype of one to many. Multicast is advantageous over unicast particularly in bandwidth reduction, but unicast is till the extensively widen communication form network [4].

\section{CONGESTION CONTROLALGORITHMS CLASSIFICATION}

The Congestion Control Algorithms are categorized largely depend on the below criterion:

- Can be classified by the category and size of the response received from the network

- Can be classified by increasing the deploy capability on the network. Just the dispatcher necessities for the alteration (or) dispatcher and destination necessitate only the router necessitate for the modification (or) tall the three: dispatcher, destination and routers needs for the modification.

- Can be classified by the equality criterion it use minimum potential delay, the proportional, and max min. 
- Can be classified by the aspect of performance. To create developments in performance: highest bandwidth $\mathrm{n} / \mathrm{w}$, lossy links, fairness, benefit to short flows, variable-rate links

Algorithms of congestion control are capable to use network attentiveness as a standard. Subsequent are three groupings for the congestion control mechanisms. The Black box comprises of a group of algorithms based on the thought that reflects on black box n/w kind, affected of no awareness of its condition much than the binary response leading to congestion. A Grey box is the grey group near to that use the dimensions to assess the accessible bandwidth and level of contention or still the conditional features of the congestion. Due to the chance of wrong assessment and measurement dimensions, a n/w is examined as grey box. A Green box comprises bimodal congestion control through that it can compute explicitly the fairs hare, also the $\mathrm{n} / \mathrm{w}$ assisted drawback, as a n/w transfer through its transport layer. So, it is considered as green box.

\section{A. Black Box}

A black box is a categorized congestion control and it's also called as the Blind Congestion Control approach and this procedure utilize the AIMD (Additive Increase Multiplicative Decrease) algorithm. AIMD implements TCP window adjustments.

\section{B. Grey Box}

Grey box is also called as congestion control with measurement based. Values of the TCP confide on the losses of packet as virtual signal of the congestion signal as the congested links There are a number of reasons for indicating the congestion one of the common reasons is the loss of packet and random bit corruption is the main cause for the packet loss and is caused when bandwidth is still accessible. At the source aspect, acknowledgement-based loss detection can altered by interweave traffic on invert path . The loss of packet, as a binary reaction, can't specify the level of rivalry earlier than the amount of congestion.

\section{Green Box}

A Green box carries bimodal congestion control mechanism with the aid of which it is able to calculate explicitly the fairs hare of the machine goes with the flow in the community. Bimodal congestion prevention and Control mechanism for each drift the fair percentage of the entire bandwidth that needs to be allotted is calculated at any point through method execution. A RED also known as (Random Early Detection) of packet that at random dropped within fraction to the router size queue, triggering the multiplicative decreasing in several flows. In ECN (Explicit Congestion Notification) routers are enabled to probabilistic mark a bit in the IP header instead of dropping packets, near intimate the end-hosts of imminent congestion when the length of the queue exceeds a threshold. The VCP (variable structure congestion control protocol) uses two ECN (Explicit Congestion Notification) bits to clearly get the feedback of the network status of congestion [5].

\section{LITERATURE SURVEY}

\begin{tabular}{|c|c|c|c|c|c|}
\hline $\begin{array}{l}\text { S } \\
\text { No }\end{array}$ & $\begin{array}{l}\text { Approach/ } \\
\text { REF }\end{array}$ & $\begin{array}{c}\text { Source of Congestion } \\
\text { Information }\end{array}$ & Strengths/ Weakness & Parameters & $\begin{array}{c}\text { Experimental } \\
\text { Result }\end{array}$ \\
\hline 1. & $\begin{array}{c}\text { Router Mechanism } \\
{[6]}\end{array}$ & $\begin{array}{l}\text { When router discovers } \\
\text { a flow which does not } \\
\text { exhibit TCP-friendly } \\
\text { behavior. }\end{array}$ & $\begin{array}{l}\text { Non-TCP friendly flows } \\
\text { can only be achieved } \\
\text { with router support. }\end{array}$ & $\begin{array}{l}\text { offered load, } \\
\text { packet drop rate, } \\
\text { bandwidth flow }\end{array}$ & $91.76 \%$ \\
\hline 2. & $\begin{array}{l}\text { TCP Reno Congestion } \\
\text { control [7] }\end{array}$ & Congestion window & $\begin{array}{c}\text { Fast retransmit } \\
\text { mechanism, Improved } \\
\text { retransmission timeout }\end{array}$ & $\begin{array}{l}\text { Send rate, Loss } \\
\text { Rate, round trip } \\
\text { time }\end{array}$ & Fair result \\
\hline 3. & \begin{tabular}{lr}
\multicolumn{3}{l}{ Switchover Mechanism } \\
of flow and Congestion \\
Control, \\
Listening \\
(RLA) [8]
\end{tabular} & $\begin{array}{l}\text { The rate-based } \\
\text { controller }\end{array}$ & $\begin{array}{l}\text { Reliable Multicast } \\
\text { communication, } \\
\text { Prevents a drop-to-zero } \\
\text { problem. }\end{array}$ & $\begin{array}{l}\text { Rate-Based, } \\
\text { NAKs, } \\
\text { Transmission rate }\end{array}$ & $92.45 \%$ \\
\hline 4. & $\begin{array}{l}\text { Source rates tend to be } \\
\text { distributed in order to } \\
\text { maximize an objective } \\
\text { function calledFAh } \\
\text { ("FAhfairness") [9] }\end{array}$ & Congestion window & $\begin{array}{l}\text { Packet loss ratios, which } \\
\text { can be expected in a } \\
\text { provide } n / w \text { with a no of } \\
\text { competing TCP or TCP- } \\
\text { friendly sources. }\end{array}$ & $\begin{array}{l}\text { Objective function } \\
\text { called FAh ("FAh } \\
\text { fairness"), RTT, } \\
\text { re-transmission } \\
\text { timeout }\end{array}$ & Fair result \\
\hline 5. & $\begin{array}{l}\text { Multicast window } \\
\text { scheme using a distinct } \\
\text { window size for each }\end{array}$ & Congestion window & \begin{tabular}{lr} 
TCP & Fairness is \\
basically & \multicolumn{2}{c}{ window } \\
oriented and that in
\end{tabular} & $\begin{array}{l}\text { Rate oriented and } \\
\text { window oriented } \\
\text { fairness, RTT }\end{array}$ & \\
\hline
\end{tabular}




\begin{tabular}{|c|c|c|c|c|c|}
\hline & $\begin{array}{l}\text { receiver, and enforcing } \\
\text { it as the limit on the } \\
\text { number of outstanding } \\
\text { packets to that } \\
\text { receiver.[10] }\end{array}$ & $\begin{array}{l}\text { order to achieve this } \\
\text { form of fairness via rate } \\
\text { based regulation; the } \\
\text { regulation algorithm } \\
\text { must explicitly depend } \\
\text { on receivers RTT. }\end{array}$ & $93.45 \%$ \\
\hline 6. & $\begin{array}{c}\text { Easay AIMD pattern } \\
\text { for unicast flows (Rate } \\
\text { Adaption Protocol } \\
\text { RAP).[11] }\end{array}$ & ACK packets and RTT & $\begin{array}{l}\text { Additional fine-grained } \\
\text { delay based congestion } \\
\text { avoidance }\end{array}$ & $\begin{array}{c}\text { RTT,TCP } \\
\text { throughput }\end{array}$ & Fair result \\
\hline 7 & $\begin{array}{c}\text { TFRC-The TCP- } \\
\text { Friendly rate control } \\
\text { Protocol.[12] }\end{array}$ & Roundtrip time & $\begin{array}{l}\text { Stable sending rate, } \\
\text { Supports additional } \\
\text { delay-based congestion } \\
\text { avoidance by adjusting } \\
\text { the inter-packet gap. }\end{array}$ & RTT, loss rate & Fair result \\
\hline
\end{tabular}

\section{PROPOSED WORK}

All the activity which we are managing by our proposed scheme is assure about the reliability aspects due to the connection oriented TCP services and the previous working of the (MCA) which stands for "multicast congestion avoidance" lower version protocol has been implemented as UDP connection-less services. Hence reliability will be increased significantly.

MAC implementation with unreliable source is not well for all those organization who especially working for confidential data communication framework all the schemes define by the computer society for network point of view is providing the resulted contents with different connection scheme TCP is always there to provides the reliable source of information . Network performance always varies with the specification of connection protocols.

\section{EXPERIMENT AND SIMULATION ENVIRONMENT}

Simulation environment of the proposed work is about the work on Ethernet 802.11 with the other Media Access Layer protocol on the simulator Wireshark which is based on the network specification with the TCP network connection.

Wireshark is a network packet analyzer. An $\mathrm{n} / \mathrm{w}$ packet analyzer will try to seize network packets and attempts to display that packet those are as unique as possible. You ought to think about an $\mathrm{n} / \mathrm{w}$ packet analyzer as a measuring tool that utilized to take a look on what is taking place inside a network cable, much like a voltmeter that is utilized through an electrician to look at what is occurring internally within an electric cable (but at a better stage, of course).

In the earlier, as tools were either very costly, proprietary, or both. Though, with the Wireshark advent, each which has modified. Wireshark is possibly exceptional open supply packet analyzers obtainable these days.

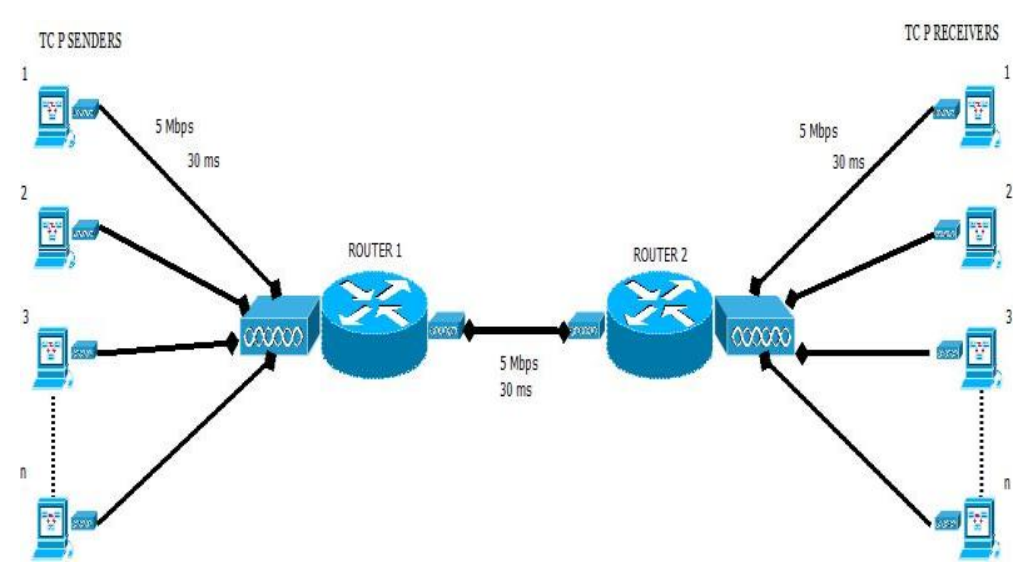

Fig. 1 Multiple flows experimental set-up for New AIMD evaluation

\section{Pseudocode:}

MAC implementation with unreliable source is not well for all those organization who especially working for confidential data communication framework all the schemes define by the computer society for network point of view is providing the resulted contents with different connection scheme TCP is always there to provides the reliable source of information. Network performance always varies with the specification of connection protocols. Here we are presenting the TCP code to for reliable connection and MAC code implementation. 
The following code uses Indy.Sockets. UDPClient to send messages to pcs on a LAN network using the TCP address and listening port (which I store in a database):

public static void SendMessage(Client pClient)

\{

TCPClienttcpClient $=$ new TCPClient();

TCPClient.Host $=$ pClient.ServerIP;

TCPClient.Port $=$ pClient.fListeningOnTCPPort;

tcpClient.SendBuffer(pClient.RestartMessage);

\}

But requirements have changed and we now need to be able to send messages using the MAC address instead (also stored in database), is it possible to send a similar message by making the use MAC address?

The MAC address on a live is the similar subnet TCP connection will be certainly in the ARP cache .On Linux, you could examine on GNS3 that how the ARP cache by looking in /proc/net/arp.

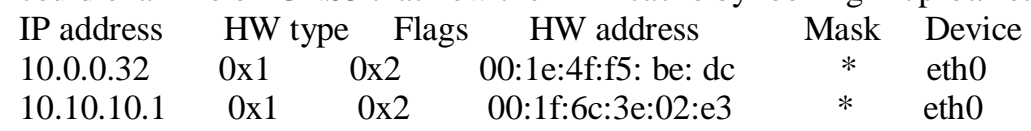

After configuring all this setting we need to bind the communication channel with interface to get the data transmission result over to the simulator that shows graphical specification of both TCP and UDP.

\section{Script for the network topology}

Save this script to a file (e.g. /usr/local/bin/chanhop.sh) and run:

\# chmod 700 /usr/local/bin/chanhop.sh

As root, to make the script executable. Running the script with no arguments displays

the following usage instructions:

chanhop.sh: Usage:

./chanhop.sh [-i|--interface] [-b|--band] [-d|--dwelltime]

-i or --interface specifies the interface name to hop

-b or --band specifies the bands to use for channel hopping, one of

IEEE80211B Channels 1-11 [default]

IEEE80211BINTL Channels 1-13

IEEE80211BJP Channels 1-14

IEEE80211A Channels 36-161

Use multiple -b arguments for multiple channels

-d or --dwelltime amount of time to spend on each channel [default .25 seconds]

e.x. ./chanhop.sh -i ath0 -b IEEE80211BINTL -b IEEE80211A -d .10 Exiting.

Starting Channel Hoping:

\#./chanhop.sh -i ath0

\# ./chanhop.sh -i ath0 -b IEEE80211B -b IEEE80211A -d .1

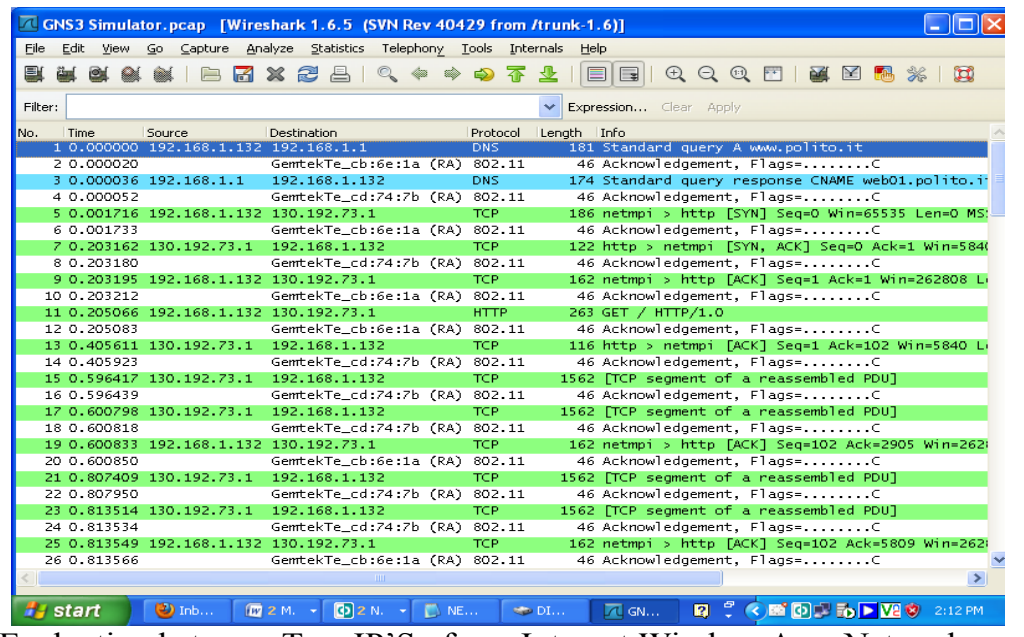

Fig. 2 Performance Evaluation between Two IP'S of any Intranet Wireless Area Network over TCP and 802.11 
To make the communication in network environment we need to consider all the 802.11 protocol series if we will take each protocol individually we found that they have performed differently with QoS specification and normally without QoS Model they give the Following result that has been got in a global network environment if we analyzing the performance specification in an intranet area network we need to gathering both 802.11 and TCP protocols parallel and then we necessity to study the performance of TCP and 802.11 protocols for an individual time session of TCP protocol with QoS specification.

\section{RESULTS ANALYSIS}

To assess the New AIMD algo, we conduct experiments based on the Wireshark simulation. The Wireshark simulation help us to evaluate the behavior of New AIMD under diverse network condition. In this section we focus on the simulation results.Figure.2shows the network topology used in the simulation. The topology is a simple dumbbell topology network. The bottleneck link is set to $5 \mathrm{Mbps}$. The links that connect the senders and the receivers to the router have bandwidth of $5 \mathrm{Mbps}$. The end-to-end RTT is set to30ms. The router queue size is 100 packets. Figure. 4,5 and 6 shows the results between two nodes for experiments with 2 flows with TCP, UDP and HTTP Protocols in the single bottleneck link respectively.

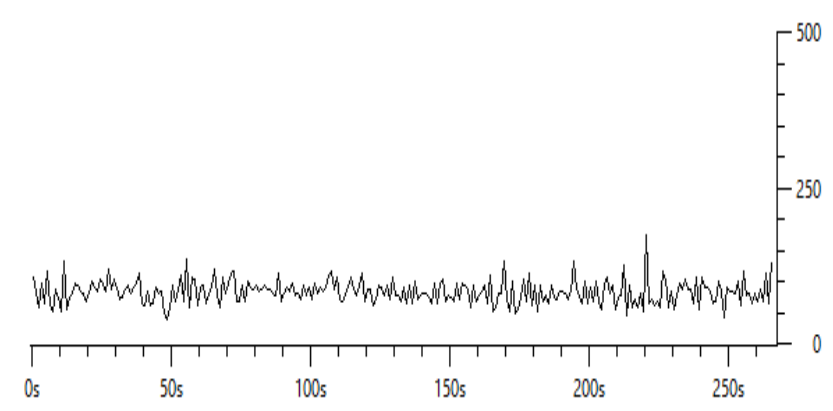

Fig 3 The result between two nodes in experiment with 2 flows

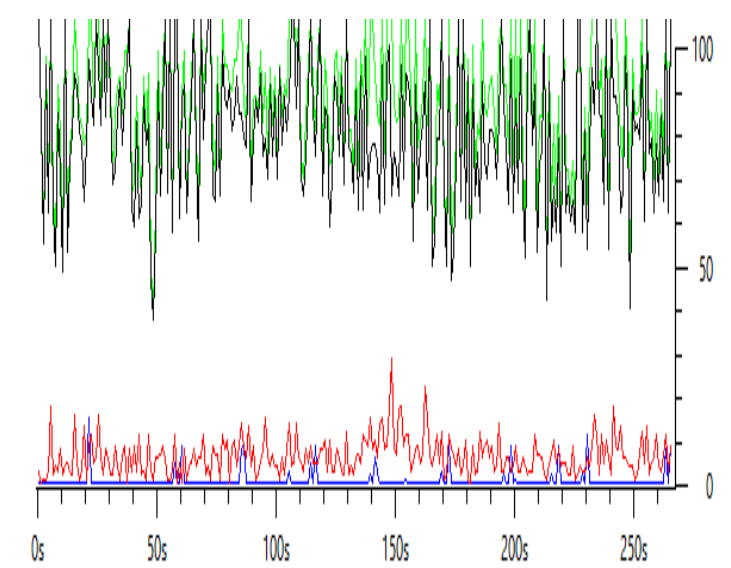

Fig. 4 The result between two nodes in experiment with 2 flows with TCP, UDP and HTTP.

Table 3 shows the comparison between the throughput and average packet loss rates for different number off low in same bottleneck link bandwidths. The throughput is calculated over a period of 300 seconds after the flow reaches steady state. Due to the large period for averaging the throughput and the buffer size of 100 packets, TCP, UDP and HTTP flow seems to be able to obtain reasonable high throughput.

Table 3: Throughput for different Protocol of 2 flow in single bottleneck link with New AIMD algorithm.

\begin{tabular}{|c|c|c|c|}
\hline \multirow[b]{2}{*}{ Protocol } & \multicolumn{3}{|c|}{2 Flow } \\
\hline & $\begin{array}{c}\text { Total Throughput per-flow } \\
\text { sent KB }\end{array}$ & $\begin{array}{c}\text { Total Throughput per-flow } \\
\text { received KB }\end{array}$ & $\begin{array}{l}\text { Rate of Throughput } \\
\text { received \% }\end{array}$ \\
\hline TCP & 84477.76 & 84477.76 & 100 \\
\hline UDP & 65368.328 & 65196.956 & 99.73 \\
\hline HTTP & 34138.592 & 34138.592 & 100 \\
\hline
\end{tabular}


The Simulator Wireshark simulates the actual performance with Throughput and Packet Delay specification assigned by QoS appraisal Model. In above intranet scenario we are considering Two IP addresses i.e. 192.168.1.132 and 130.192.73.1 both of the IP'S will be in communication through TCP whenever anyone ip will interact with other ip , QoS standards 802.11 will always there for providing services with defined QoS specification. The sequences of request promoting at sequential timer set and performing the acknowledgements after getting response shows that req1,req2,req3,req $4 \ldots \ldots \ldots \ldots . .$. .req $\mathrm{N}$. Has been performing completely without interruption, here we call for the connection with time session 0.000000 to 0.813566 that evaluates all the request has been done without delay . It means Our proposed QoS model has been working properly without data loss and also providing efficiency specification for any limited communication area network all the devices are co-ordinate with each other in any network topology with such a wonderful mechanism, connection has been established by TCP protocol that support buffering at both end so that stream delivery services support audio, video and multimedia communication.

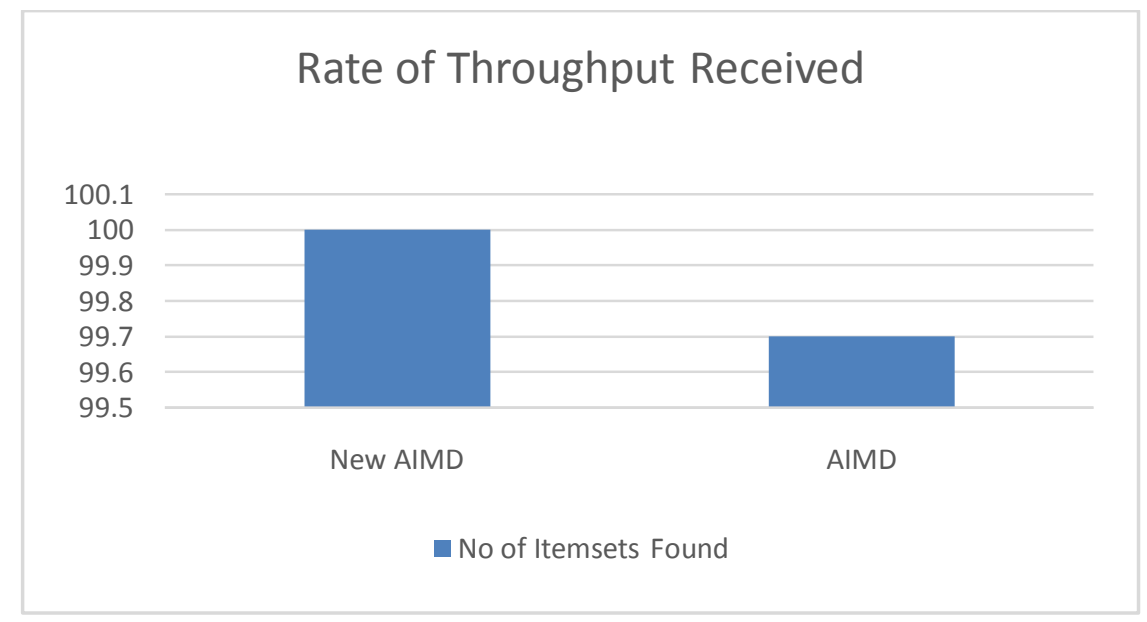

Fig. 5 Through put Performance between AIMD and New Method

\section{CONCLUSION}

QoS Model contains TCP and IEEE protocols therefore it expansive to implement but economical for all the private and government organization that provides reliable network for communication. when TCP connection has been established between nodes for session $1.988 \mathrm{sec}$ simulator captured 140 ,all the 140 packets has been acknowledged and display shows 140 i.e. no packet lost or ignored during whole session, all the packets has been captured in 1.9sec that is the best time case complexity for transmission of data in any intranet system. The QoS model is working successfully.

\section{REFERENCES}

[1] J. W. Chung, "Congestion control for streaming media," Ph. D. dissertation, Polytechnic Inst., Worcester, 2005.

[2] Sally Floyd and Kevin Fall, "Promoting the use of end-to-end congestion control in the Internet," IEEE/ACM Transactions on Networking, vol. 7, no. 4, pp. 458-472, Aug. 1999.

[3] B. Braden, D. Clark, J. Crowcroft, B. Davie, S. Deering, D. Estrin, S. Floyd, V. Jacobson, G. Minshall, C. Partridge, L. Peterson, K. Ramakrishnan, S. Shenker, J.Wroclawski, and L. Zhang, "RFC 2309: Recommendations on queue management and congestion avoidance in the Internet," Apr. 1998, Status: INFORMATIONAL

[4] B. Subramani, Dr. T. Karthikeyan, "A Review on Congestion Control”, ISSN (Print) : 2319-5940, Vol. 3, Issue 1, January 2014, IJARCCE.

[5] Mahammad Mastan, P.Suresh Verma, Mohammed Ali Hussain, "A SURVEY ON CONGESTION CONTROL MECHANISMS", eISSN: 2319-1163,Volume: 03 Issue: 09 | Sep-2014, IJRET

[6] S. Floyd and K. Fall, "Promoting the Use of End-to-end Congestion Control in the Internet," IEEE/ACM Trans. Net., vol. 7, no. 4, Aug. 1999, pp. 458-72.

[7] J. Padhye et al., "Modeling TCP Reno Performance: A Simple Model and Its Empirical Validation," IEEE/ACM Trans. Net., vol. 8, no. 2, Apr. 2000, pp. 133-45.

[8] H. A. Wang and M. Schwartz, "Achieving Bounded Fairness for Multicast and TCP Traffic in the Internet," Proc. ACM SIGCOMM, 1998.

[9] M. Vojnovic, J. Y. Le Boudec, and C. Boutremans, “Global Fairness of Additive-Increase and Multiplicative-Decrease with Heterogeneous Round-Trip Times," Proc. IEEEa INFOCOM 2000, Tel Aviv, Israel, Mar. 2000.

[10] S. J. Golestani and K. K. Sabnani, "Fundamental Observations on Multicast Congestion Control in the Internet," Proc. INFOCOM '99, Mar. 1999, vol. 2, pp. 990- 1000.

[11] R. Rejaie, M. Handley, and D. Estrin, "Rap: An End-to-End Rate-Based Congestion Control Mechanism for Realtime Streams in the Internet," Proc. IEEE INFOCOM,Mar. 1999.

[12] S. Floyd et al., "Equation-based Congestion Control for Unicast Applications," Proc. ACM SIGCOMM, Stockholm, Sweden, Aug. 2000, pp. 43-56. 